\title{
The co-existence of hot and cold gas in debris discs (Corrigendum)
}

\author{
I. Rebollido ${ }^{1}$, C. Eiroa ${ }^{1}$, B. Montesinos ${ }^{2}$, J. Maldonado ${ }^{3}$, E. Villaver ${ }^{1}$, O. Absil ${ }^{4}$, A. Bayo ${ }^{5,6}$, H. Canovas ${ }^{1,7}$, \\ A. Carmona ${ }^{8}$, Ch. Chen ${ }^{9}$, S. Ertel ${ }^{10}$, A. Garufi ${ }^{1}$, Th. Henning ${ }^{11}$, D. P. Iglesias ${ }^{5,6}$, R. Launhardt ${ }^{11}$, R. Liseau ${ }^{12}$, \\ G. Meeus ${ }^{1}$, A. Moór ${ }^{13}$, A. Mora ${ }^{14}$, J. Olofsson ${ }^{5,6}$, G. Rauw ${ }^{4}$, and P. Riviere-Marichalar ${ }^{15}$ \\ ${ }^{1}$ Departamento Física Teórica, Universidad Autónoma de Madrid, Cantoblanco, 28049 Madrid, Spain \\ e-mail: isabel.rebollido@uam.es \\ ${ }^{2}$ Centro de Astrobiología (CAB, CSIC-INTA), ESAC Campus, Camino Bajo del Castillo s/n, Villanueva de la Cañada, 28692 Madrid, \\ Spain \\ ${ }^{3}$ INAF, Osservatorio Astronomico di Palermo, Piazza del Parlamento 1, 90134 Palermo, Italy \\ ${ }^{4}$ STAR Institute, Université de Liège, F.R.S.-FNRS, 19c Allée du Six Août, 4000 Liège, Belgium \\ ${ }^{5}$ Instituto de Física y Astronomía, Facultad de Ciencias, Universidad de Valparaíso, Casilla, 5030 Valparaíso, Chile \\ ${ }^{6}$ Núcleo Milenio de Formación Planetaria-NPF, Universidad de Valparaíso, Av. Gran Bretaña, 1111 Valparaíso, Chile \\ ${ }^{7}$ European Space Astronomy Centre (ESA), PO Box 78, Villanueva de la Cañada, 28691 Madrid, Spain \\ ${ }^{8}$ Université de Toulouse, UPS-OMP, IRAP, 31400 Toulouse, France \\ ${ }^{9}$ Space Telescope Science Institute, 3700 San Martin Drive, Baltimore, MD 21212, USA \\ 10 Steward Observatory, Department of Astronomy, University of Arizona, Tucson, AZ 85721, USA \\ ${ }^{11}$ Max-Planck-Institut für Astronomie (MPIA), Königstuhl 17, 69117 Heidelberg, Germany \\ 12 Department of Space, Earth and Environment, Chalmers University of Technology, Onsala Space Observatory, 43992 Onsala, \\ Sweden \\ ${ }^{13}$ Konkoly Observatory, Research Centre for Astronomy and Earth Sciences, PO Box 67, 1525 Budapest, Hungary \\ ${ }^{14}$ Aurora Technology B.V. for ESA, ESA-ESAC, Villanueva de la Cañada, 28691 Madrid, Spain \\ 15 Instituto de Ciencia de Materiales de Madrid (CSIC), Calle Sor Juana Inés de la Cruz 3, 28049 Cantoblanco, Madrid, Spain
}

A\&A, 614, A3 (2018), https : //doi . org/10 . 1051/0004-6361/201732329

Key words. stars: general - planetary systems - comets: general - ISM: clouds - circumstellar matter - errata, addenda

Due to a bad correction of telluric lines some $\mathrm{Na}$ I absorptions detected towards some stars were not mentioned in the original paper. This corrigendum presents a proper telluric subtraction, carried out with MOLECFIT ${ }^{1}$ (Smette et al. 2015, Kausch et al. 2015).

Tables 3 and 4 from the original paper are rectified below with the corrected $\mathrm{Na}$ I absorptions shown in boldface text.

Figure 4 of the original paper showed an incorrect plot of the $\mathrm{Na}$ I D line features observed towards HD 138813. Figure 1 is the corrected version of the original paper's Fig. 4.

Below we summarize the corrected $\mathrm{Na}$ I absorptions of each concerned star:

- HD 9672: the Na I D absorption at $11.5 \mathrm{~km} \mathrm{~s}^{-1}$ coincides with the Ca II feature and the radial velocity of the ISM.

- HD 131488: besides the already reported non-photospheric absorptions at $\sim 5.3 \mathrm{~km} \mathrm{~s}^{-1}$, a Na I D2 feature at $-13.9 \mathrm{~km} \mathrm{~s}^{-1}$ is detected. A similar feature is detected towards field stars and most likely has an interstellar medium origin.

- HD 138813: two new non-photospheric features are detected in the $\mathrm{Na}$ I D lines (see Fig. 1). The approximately $-22.2 \mathrm{~km} \mathrm{~s}^{-1}$ absorption is close to the Ca II $\mathrm{K}$ nonphotospheric absorption and the G ISM cloud; thus, an interstellar origin is more plausible. A feature in emission at $0.1 \mathrm{~km} \mathrm{~s}^{-1}$ is detected in both $\mathrm{Na} \mathrm{I} \mathrm{D}$ lines at the radial velocity of the star; this feature is related to the star.

1 https://www.eso.org/sci/software/pipelines/skytools/ molecfit

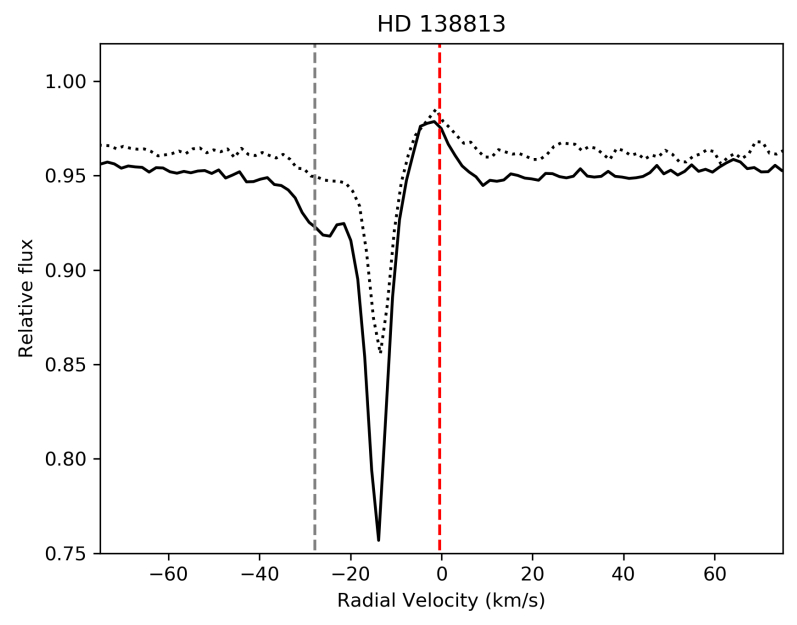

Fig. 1. Figure corrected for HD $138813 \mathrm{Na}$ I D2 (black solid line) and D1 (black dotted line) lines. The radial velocities of the star (red dashed line) and of the ISM (grey dashed line) are also shown. Three Na I features are present, including an emission line at the radial velocity of the star.

- HD 156623: an emission Na I D feature is present at the radial velocity of the star. Figure 2 shows the emission feature in both Na I D lines.

- HD 172555: two narrow non-photospheric absorptions are detected. One of them, at approximately $-20.4 \mathrm{~km} \mathrm{~s}^{-1}$ is close to the $\mathrm{G}$ cloud, and therefore possibly of ISM 
Table 3. Radial velocities and equivalent widths (EWs) of the Ca II H\&K and Na I D non-photospheric absorptions, radial velocities of the stars, and projected velocities of ISM clouds in the Colorado model.

\begin{tabular}{|c|c|c|c|c|c|c|c|}
\hline \multicolumn{8}{|c|}{ Stars with non-photospheric absorptions } \\
\hline Star & $\begin{array}{r}v_{\text {CaIIK }} \\
\left(\mathrm{km} \mathrm{s}^{-1}\right) \\
\end{array}$ & $\begin{array}{l}\mathrm{EW}(\mathrm{K} / \mathrm{H}) \\
\mathrm{m \AA}\end{array}$ & $\begin{array}{l}\Delta v \text { CaIIK } \\
\left(\mathrm{km} \mathrm{s}^{-1}\right) \\
\end{array}$ & $\begin{array}{l}v_{\mathrm{NaID}} \\
\left(\mathrm{km} \mathrm{s}^{-1}\right)\end{array}$ & $\begin{array}{r}\mathrm{EW}(\mathrm{D} 2 / \mathrm{D} 1)^{(c)} \\
\mathrm{m \AA} \\
\end{array}$ & $\begin{array}{l}v_{\text {rad }} \\
\left(\mathrm{km} \mathrm{s}^{-1}\right)\end{array}$ & $\begin{array}{r}v_{\text {ISM }}{ }^{(d)} \\
\left(\mathrm{km} \mathrm{s}^{-1}\right) \\
\end{array}$ \\
\hline HD $9672^{(a)}$ & $13.5 \pm 2.3$ & $10.6 / 6.0$ & 7.4 & $11.5 \pm 2.3$ & 16.4/9.3 & $11.0 \pm 2.0$ & 11.01 (LIC) \\
\hline HD $32297^{(a)}$ & $22.7 \pm 3.0$ & $19.4 / 8.9$ & 9.7 & $23.8 \pm 2.5$ & $93.0 / 84.0$ & $19.7 \pm 2.0$ & 23.59 (LIC) \\
\hline \multirow{2}{*}{ HD $110058^{(a)}$} & $12.7 \pm 3.3$ & $27.0 / 24.1$ & 6.8 & $12.5 \pm 2.8$ & $46.0 / 39.1$ & $12.6 \pm 2.4$ & $-14.46(\mathrm{G})$ \\
\hline & $1.2 \pm 3.3$ & $19.9 / 10.0$ & 12.6 & $0.9 \pm 2.8$ & $34.0 / 18.9$ & & \\
\hline \multirow[t]{2}{*}{ HD $131488^{(a)}$} & $4.0 \pm 2.7$ & $5.7 /-$ & 9.6 & $5.3 \pm 2.2$ & $42.1 / 34.2$ & $5.8 \pm 1.5$ & $-23.91(\mathrm{G})$ \\
\hline & $-3.2 \pm 2.7$ & $4.0 /-$ & 4.2 & $-13.88 \pm 2.2$ & 7.2/- & & \\
\hline \multirow[t]{3}{*}{ HD $131835^{(a)}$} & $2.2 \pm 2.7$ & $0.7 /-$ & 2.3 & - & $<0.9$ & $2.6 \pm 1.4$ & 18.56 (GEM) \\
\hline & $-5.9 \pm 2.7$ & $2.1 / 1.4$ & 7.9 & - & $<0.9$ & & \\
\hline & $-13.8 \pm 2.7$ & $8.3 / 4.1$ & 7.4 & $-11.8 \pm 2.0$ & $41.0 / 32.1$ & & \\
\hline \multirow[t]{3}{*}{ HD 138813} & $-27.5 \pm 3.0$ & $5.6 /-$ & 18.7 & $-22.2 \pm 2.2$ & $5.9 / 3.1$ & $-0.4 \pm 2.0$ & $-27.82(\mathrm{G})$ \\
\hline & & & & $-12.0 \pm 2.5$ & $18.0 / 15.4$ & & \\
\hline & & & & $0.1 \pm 2.0$ & $-7.55 /-5.2$ & & \\
\hline \multirow[t]{2}{*}{ HD 146897} & $-8.0 \pm 2.7$ & $12.4 /-$ & 7.2 & $-8.8 \pm 2.1$ & $118.2 / 80.0$ & $-4.0 \pm 1.5$ & $-29.19(\mathrm{G})$ \\
\hline & $-30.0 \pm 2.7$ & $5.9 /-$ & 4.6 & - & $<0.8$ & & \\
\hline \multirow[t]{2}{*}{ HD $156623^{(a)}$} & $-15.0 \pm 2.7$ & $9.0 / 3.7$ & 18.7 & $-1.8 \pm 2.6$ & $-10.6 /-8.5$ & $-0.2 \pm 1.5$ & $-24.89(\mathrm{G})$ \\
\hline & $8.1 \pm 2.7$ & $4.3 /-$ & $6.0^{(e)}$ & - & $<0.7$ & & \\
\hline HD $172555^{(a)}$ & $2.3 \pm 3.0$ & $9.9 / 4.0$ & 9.7 & $\begin{array}{l}15.3 \pm 2.1 \\
-20.4 \pm 2.1\end{array}$ & $\begin{array}{r}8.0 /- \\
3.2 / 1.8\end{array}$ & $2.6 \pm 1.9$ & $-16.84(\mathrm{G})$ \\
\hline HD $181296^{(b)}$ & $-22.8 \pm 3.8$ & $3.9 / 2.2$ & 8.4 & $-22.9 \pm 2.4$ & $4.0 /-$ & $-3.0 \pm 3.0$ & $-18.51(\mathrm{G})$ \\
\hline
\end{tabular}

Notes. The FWHM velocity dispersion of the Ca II K lines are also given. ${ }^{(a)}$ Stars with FEBs or red wings. ${ }^{(b)}$ The line of sight towards this star does not traverse any ISM cloud in the Colorado model but it passes within $20^{\circ}$ of several clouds. Among these, cloud $\mathrm{G}$ is the closest one in velocity. ${ }^{(c)}$ Upper limits are shown for those stars without Na non-photospheric absorption. ${ }^{(d)}$ Names within brackets are ISM clouds in the Colorado model (Redfield \& Linsky 2008). ${ }^{(e)}$ The velocity dispersion for this absorption is highly variable, and the value shown here is the value obtained from the median spectra.

Table 4. Summary of the non-photospheric absorption features of the observed cold gas-bearing debris disc stars.

\begin{tabular}{|c|c|c|c|c|c|c|c|}
\hline \multicolumn{8}{|c|}{ Stars with non-photospheric absorptions } \\
\hline \multirow[b]{2}{*}{$\underline{\text { Star }}$} & \multicolumn{3}{|c|}{$\mathrm{Ca}$ II } & \multicolumn{3}{|c|}{$\mathrm{Na} \mathrm{I}$} & \multirow[t]{2}{*}{ Angle (deg.. $)^{(c)}$} \\
\hline & $V_{\text {star }}$ & $V_{\text {ISM }}$ & Neither & $V_{\text {CaII }}$ & $V_{\text {star }}$ & $\overline{V_{\text {ISM }}}$ & \\
\hline HD $9672^{(a)}$ & Y & Y & & $\mathbf{Y}$ & $\mathbf{Y}$ & & $70-90(1,2,3)$ \\
\hline HD $32297^{(a)}$ & $\mathrm{Y}$ & $\mathrm{Y}$ & & $\mathrm{Y}$ & $\mathrm{Y}$ & & $80-88(4,5)$ \\
\hline HD $110058^{(a)}$ & $\mathrm{Y}$ & & $\mathrm{Y}$ & $\mathrm{Y}$ & $\mathrm{Y}$ & & $\sim 90(6)$ \\
\hline HD $131488^{(a)}$ & Y & Y & & Y & $\mathrm{Y}$ & & $82(7)$ \\
\hline HD $131835^{(a)}$ & Y & Y & & Y & & & $75-83(8,9,14)$ \\
\hline HD 138813 & & $\mathrm{Y}$ & & $\mathbf{Y}$ & $\mathbf{Y}^{(b)}$ & $\mathrm{Y}$ & $32(10)$ \\
\hline HD 146897 & Y & Y & & $\mathrm{Y}$ & $\mathrm{Y}$ & & $84(11)$ \\
\hline HD $156623^{(a)}$ & & & $\mathrm{Y}$ & & $\mathbf{Y}^{(b)}$ & & 34 (10) \\
\hline HD $172555^{(a)}$ & $\mathrm{Y}$ & & & & & $\mathbf{Y}$ & 75 (12) \\
\hline HD 181296 & & & $\mathrm{Y}$ & $\mathbf{Y}$ & & & $>70(13)$ \\
\hline
\end{tabular}

Notes. Columns 2-4: coincidences of the Ca II velocity components with the radial velocity of the star, Colorado model ISM clouds along the line of sight, or none of both. Columns 5-7: coincidences of the Na I velocity components with Ca II features, the radial velocity of the star, or the ISM. Column 8 gives the inclination angle of the debris disc associated with the stars. Empty spaces mean non-detections. ${ }^{(a)}$ Stars with FEBs or red wings. ${ }^{(b)}$ Features in emission.

References. ${ }^{(c)}$ References for inclination angle: (1) Hughes et al. (2008); (2) Moór et al. (2015b); (3) Choquet et al. (2017); (4) Schneider et al. (2005); (5) Asensio-Torres et al. (2016); (6) Kasper et al. (2015); (7) Moór et al. (2017); (8) Hung et al. (2015); (9) Moór et al. (2015a); (10) Hales et al. (in prep.); (11) Thalmann et al. (2013); (12) Smith et al. (2012); (13) Smith et al. (2009); (14) Feldt et al. (2017).

origin. This feature was also reported by Kiefer et al. (2014). A weaker broad, variable feature might have been detected at $\sim 15.3 \mathrm{~km} \mathrm{~s}^{-1}$. It does not coincide with the Ca II absorption, the radial velocity of the star or the $\mathrm{G}$ cloud velocity vector. If confirmed, it would have a circumstellar origin.
- HD 181296: the absorption detected in Na I D2 coincides with the one observed in Ca II.

The only remarkable change in the results with respect to the original paper is the Na I emission towards HD 138813 and HD 156623. However, the analysis of those emissions are beyond 
I. Rebollido et al: The co-existence of hot and cold gas in debris discs (Corrigendum)

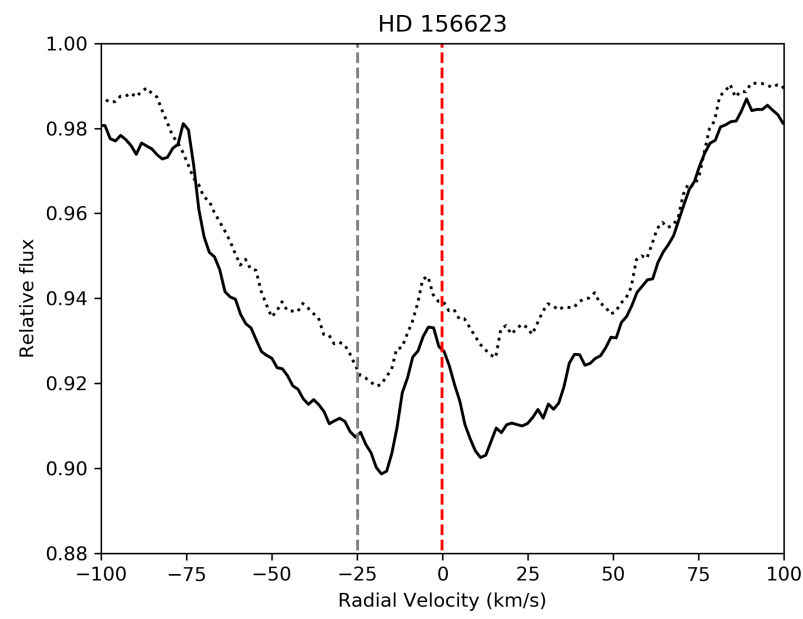

Fig. 2. Figure for HD 156623 showing the emission feature at the radial velocity of the star in both $\mathrm{Na}$ I D lines. The D2 component is shown in solid line while D1 is plotted in dotted line. The radial velocities of the star (red dashed line) and of the ISM (grey dashed line) are also shown. the scope of the original paper, and we emphasize that the main conclusions are not affected.

\section{References}

Asensio-Torres, R., Janson, M., Hashimoto, J., et al. 2016, A\&A, 593, A73 Choquet, É., Milli, J., Wahhaj, Z., et al. 2017, ApJ, 834, L12

Feldt, M., Olofsson, J., Boccaletti, A., et al. 2017, A\&A, 601, A7

Hughes, A. M., Wilner, D. J., Kamp, I., \& Hogerheijde, M. R. 2008, ApJ, 681, 626

Hung, L.-W., Fitzgerald, M. P., Chen, C. H., et al. 2015, ApJ, 802, 138

Kasper, M., Apai, D., Wagner, K., \& Robberto, M. 2015, ApJ, 812, L33

Kausch, W., Noll, S., Smette, A., et al. 2015, A\&A, 576, A78

Kiefer, F., Lecavelier des Etangs, A., Augereau, J.-C., et al. 2014, A\&A, 561, L10

Moór, A., Henning, T., Juhász, A., et al. 2015a, ApJ, 814, 42

Moór, A., Kóspál, Á., Ábrahám, P., et al. 2015b, MNRAS, 447, 577

Moór, A., Curé, M., Kóspál, Á., et al. 2017, ApJ, 849, 123

Redfield, S., \& Linsky, J. L. 2008, ApJ, 673, 283

Schneider, G., Silverstone, M. D., \& Hines, D. C. 2005, ApJ, 629, L117

Smette, A., Sana, H., Noll, S., et al. 2015, A\&A, 576, A77

Smith, R., Churcher, L. J., Wyatt, M. C., Moerchen, M. M., \& Telesco, C. M. 2009, A\&A, 493, 299

Smith, R., Wyatt, M. C., \& Haniff, C. A. 2012, MNRAS, 422, 2560

Thalmann, C., Janson, M., Buenzli, E., et al. 2013, ApJ, 763, L29 